\title{
Image Segmentation using Morphological Operations
}

\author{
Diya Chudasama \\ Dept. of Information \\ Technology. \\ SVMIT Engineering College \\ Bharuch,India.
}

\author{
Tanvi Patel \\ Dept. of Information \\ Technology. \\ SVMIT Engineering College \\ Bharuch,India.
}

\author{
Shubham Joshi \\ Dept. of Information \\ Technology. \\ SVMIT Engineering College \\ Bharuch,India
}

\author{
Ghanshyam I. Prajapati \\ Dept. of Information Technology. \\ SVMIT Engineering College \\ Bharuch,India
}

\begin{abstract}
Image Segmentation plays vital role in Computer Vision and Digital Image Processing. It is the process of separating the digital image into distinct region(s) possessing homogeneous properties. The main objective of image segmentation is to extract various features of the image that are used for analyzing, interpretation and understanding of images. Image segmentation is applied in various applications like medical imaging, shape detection, content-based image retrieval, robot vision, etc. Several techniques have been developed for image segmentation such as pixel-based segmentation, edge based segmentation and region based segmentation. In this paper, segmentation technique is defined using the edge detection and morphological operations. Edge detection is done using Fuzzy Canny method for better output. After detecting the edges of image, segmentation is done using morphological operation. This gives better results.
\end{abstract}

\section{General Terms}

Dilation, Morphology, Erosion, Flood Fill.

\section{Keywords}

Edge detection, Fuzzy Canny, Segmentation, Morphology, Dilation, Erosion, Flood fill.

\section{INTRODUCTION}

An image is basically two dimensional signal defined by mathematical function, $\mathrm{F}(\mathrm{x}, \mathrm{y})$ where $\mathrm{x}$ and $\mathrm{y}$ gives value of horizontal and vertical co-ordinates. Digital image processing[1] deals with system that perform various operation on digital image to improve the quality of the image by removing noise and unwanted pixels and to obtain intentional information from an image. Image segmentation is a key step in digital image processing that subdivides an image into its constituent region or object that share homogeneous attributes [2]. The main purpose of the segmentation process is to get more information in the region of interest in an image which helps in annotation of the object scene [3].

Image segmentation fundamentally works on two properties [2]:

- Discontinuity: Division of the digital image predicated due to sudden changes on intensity. For example, edge detection, point detection and line detection.
- Similarity: Dividing the digital image into region based on predicated on set of predefined criteria. For example, thresholding, region growing, region splitting and merging.

The image segmentation methods are categorized as [4]:

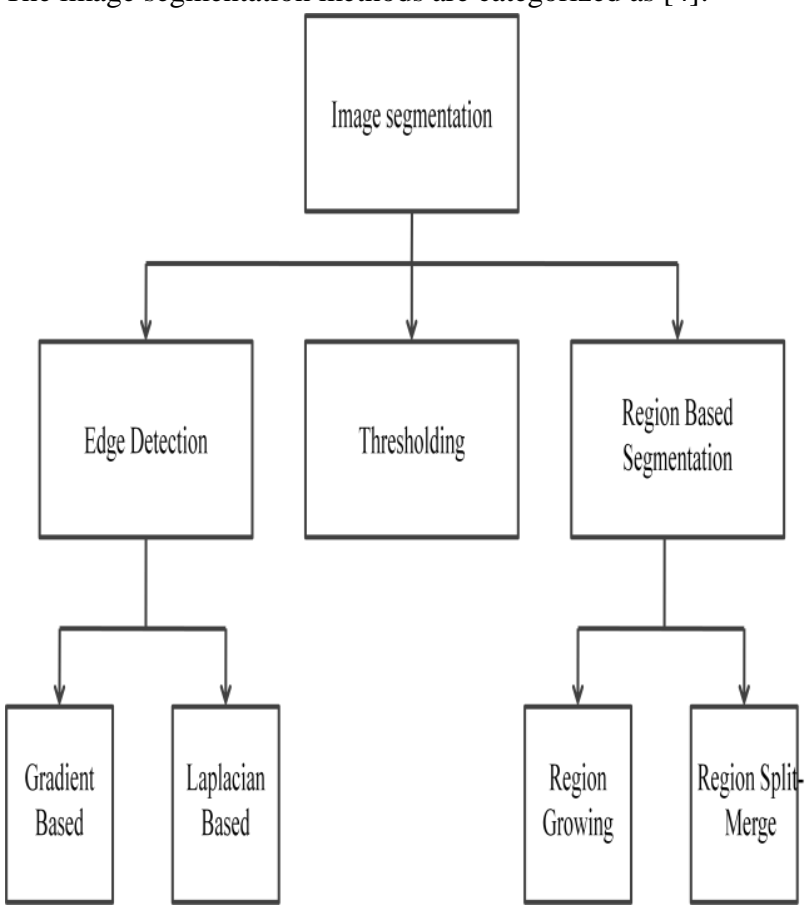

Fig 1: Image Segmentation Methods

An edge is defined as boundaries of objects or sudden change in an image which is not in a continuous form that helps to detect and identify the objects in a given image [5]. The main aim behind edge detection $[6,7]$ method is to identify and locate the points in a digital image at which intensity of the image changes. Among various technique of various edge detection technique Canny [8] operator gives better output than Sobel [9], Prewitt [10], and Laplacian method.

In Thresholding method grayscale value of the image is checked out with predefined value of the threshold. If grayscale value of the input pixel is large then output value of that pixel becomes 1 or else 0 .

$$
1, \quad \text { if } f(x, y)>T
$$




$$
0, \quad \text { if } f(x, y) \leq T
$$

There are two types of thresholding:

- Global Thresholding: Segment out the whole image with a unique threshold value.

- Local Thresholding: Segment out the image into sub-images and each sub-image has an individual threshold value.

Region Based segmentation methods divide an image into region having similar characteristic like color, texture etc. Region Growing algorithm performs a segmentation of an image with examine the neighboring pixels of a set of points, known as seed points, and determine whether the pixels could be classified to the cluster of seed point or not [11]. In method of region Split-merge [12] whole Image which is considered as a seed region splitting out into quadrant until the homogenous sub region is obtained, after the process of Splitting Merging process merge two adjacent regions according to similar characteristic.

\section{PROPOSED SEGMENTATION APPROACH}

In this paper, new approach is proposed and implemented for image segmentation. This approach suggests two phases for segmentation. First phase deals with edge detection of image with combined Fuzzy and Canny approach [13] and second concerns about morphological operation. This proposed has been implemented and the better results have been obtained for Image Segmentation. The next sub-sections discuss about this proposed approach. The basic flow is shown in figure 1 and the same is explained in next coming sections with its results.

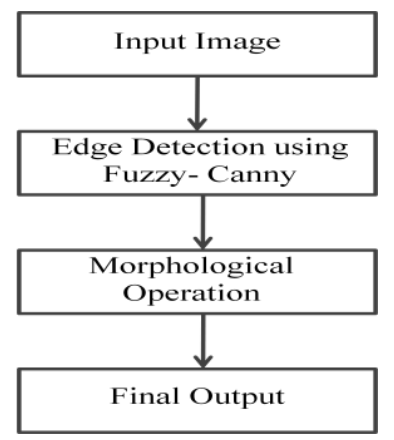

Fig 2: Flow of Proposed System

\subsection{Edge Detection using Hybrid Fuzzy- Canny method}

The hybrid of Fuzzy and Canny edge detection technique is very important method to find edges as using this method we can achieve the advantage of fuzzy approach as well as canny method. This approach gives improved results than traditional canny edge detection technique [14]. In this method first fuzzy rule based inference system [14] applied on the input image to get fuzzyfied image.

\begin{tabular}{|l|l|}
\hline $\mathbf{p 1}(\mathbf{i}, \mathbf{j})$ & $\mathrm{p} 2(\mathrm{i}+1, \mathrm{j})$ \\
\hline $\mathrm{p} 3(\mathrm{i}, \mathrm{j}+1)$ & $\mathrm{p} 4(\mathrm{i}+1, \mathrm{j}+1)$ \\
& \\
\hline
\end{tabular}

Fig 3: 2*2 Window Mask

In this approach we use $2 * 2$ window mask is used for scanning of the input image. This same method can be applied using $3 * 3$ window mask as well as combined $2 * 2$ and $3 * 3$ window mask $[15,16]$. The output retrieved from fuzzy inference system is taken as an input of the canny algorithm that gives final fuzzy-canny output. Below image shows the output of edge detected image.

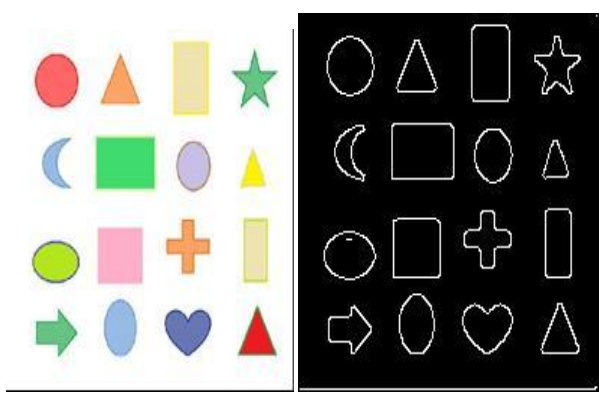

(a) Original image

(b) Fuzzy-Canny image Fig 4: Edge Detected Image

\subsection{Morphological Operations based Segmentation}

Binary images may contain countless defects. In some circumstances binary regions constructed by simple thresholding are buckled by noise and textures. Morphology is a vast extent of image processing operations that modifies the images based on shapes. It is considered to be one of the data processing methods useful in image processing. It has many applications like texture analysis, noise elimination, boundary extraction etc [17]. Morphological image processing follows the goal of eliminating all these defects and maintaining structure of image. Morphological operations are confident only on the associated ordering of pixel values, rather than their numerical values, so they are focused more on binary images, but it can also be applied to grayscale images such that their light transfer functions are unknown and thus their absolute pixel values are not taken into consideration [18].

Morphological techniques verify the image with a small template called structuring element. This structuring element is applied to all possible locations of the input image and generates the same size output. In this technique the output image pixel values are based on similar pixels of input image with is neighbors. This operation produces a new binary image in which if test is successful it will have non-zero pixel value at that location in the input image. There are various structuring element like diamond shaped, square shaped, cross shaped etc.

The base of the morphological operation is dilation, erosion, opening, closing expressed in logical AND, OR notation and described by set analysis. Among them in this paper only two operations are used dilation and erosion. Dilation adds pixels while erosion removes the pixels at boundaries of the objects. This removal or adding of pixels depends on the structuring element used for processing the image. 


\subsubsection{Dilation}

Dilation is one of the basic operators in mathematical morphology. It is applied to binary image but can also be applied to grayscale image. Dilation causes the objects to grow in size. The effect of this operation will gradually increase the boundaries of foreground pixels, thus areas grow in size and holes in that region become smaller [19].

Dilation takes two parts as data. First one is the input image to be dilated and second is the structuring element also known as kernel. With the help of this structuring element only it determines how much the image is to be dilated. The mathematical definition of dilation can be as follows [1]:

Suppose A be a set of input image coordinates and B be a set of structuring element coordinates and $\mathrm{Bx}$ is a translation of $\mathrm{B}$ so that its origin is at $\mathrm{x}$. Thus dilation of $\mathrm{A}$ by $\mathrm{B}$ is set of all points of $\mathrm{x}$ such that intersection of $\mathrm{Bx}$ with $\mathrm{A}$ is not null. In terms of set operations dilation of A by B is defined as [1][20][21]:

$$
A \oplus B=\left\{x \mid(\hat{B})_{x} \cap A \neq \phi\right\}
$$

Below image shows the output of diluted image on edge detected image output:

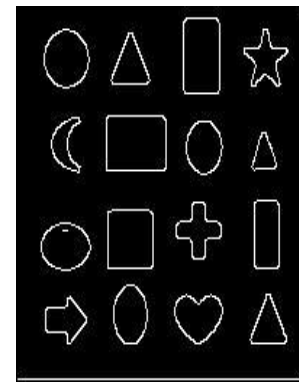

(a) Fuzzy-Canny image

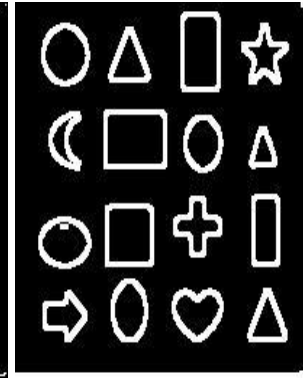

(b) Diluted image
Fig 5: Dilation image

\subsubsection{Filling the region}

Dilation operation makes the boundaries of the object thick so for segmenting the object the next step is to fill the holes. The flood fill operation is most commonly known to fill the holes in the given input image. For binary images, it basically changes the background pixels to foreground pixels until it reaches the object boundaries and for grayscale images it makes the intensity level same i.e. it makes the dark areas surrounded by lighter areas to same intensity levels [2].

In binary images and gray-scale images the boundaries of the objects need to be specified by connectivity. In binary images the starting point for filling can also be specified. If we specify holes as an argument then it is of no need to specify any starting points [2]. In this paper fill operation is used on binary image with arguments holes so it automatically fills the holes of different objects in image.

Below image shows the flood fill image on diluted image output:

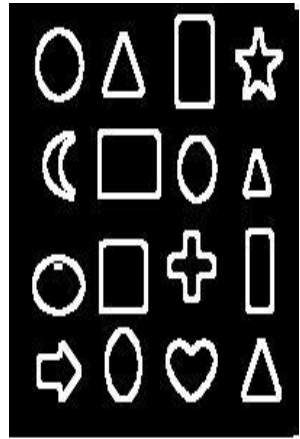

(a) Diluted image

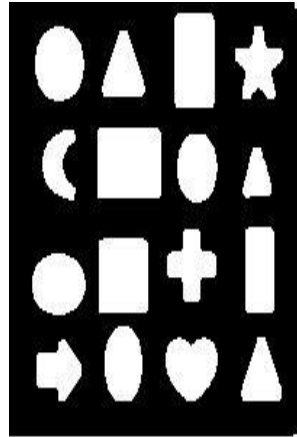

(b) Flood filled image
Fig 6: Flood fill image

\subsubsection{Erosion}

Erosion is also one of the basic operators in mathematical morphology. Erosion causes the objects to shrink or become thin in size. Erosion basically erodes away the boundaries of the foreground which results in areas of those pixels shrink in size and holes of those areas become larger [19]. So, after dilution and filling the holes of object in some images the boundaries get mixed up so to somewhat separate the boundaries erosion is applied so as to make the boundaries of the objects thinner for better output.

Erosion like same dilation takes two parts as data. First one is the input image to be eroded and second is the structuring element. With the help of this structuring element only it determines how much the image is to be eroded. The mathematical definition of erosion can be as follows [1]:

Suppose A be a set of input image coordinates and $\mathrm{B}$ be a set of structuring element coordinates and $\mathrm{Bx}$ is a translation of $\mathrm{B}$ so that its origin is at $\mathrm{x}$. Thus dilation of $\mathrm{A}$ by $\mathrm{B}$ is set of all points of $\mathrm{x}$ such that $\mathrm{Bx}$ is a subset of $\mathrm{A}$. In terms of set operations erosion of A by B is defined as [1][20][21]:

$$
A \Theta B=\left\{x \mid(B)_{x} \cap A^{c} \neq \phi\right\}
$$

Below image shows the eroded image on flood fill image output.

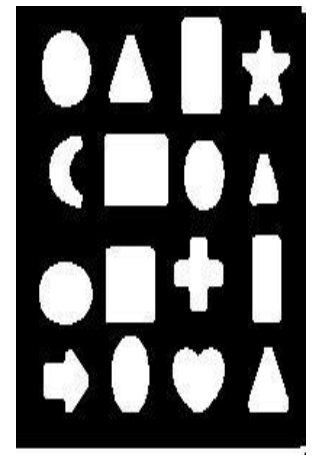

(a) Flood filled image

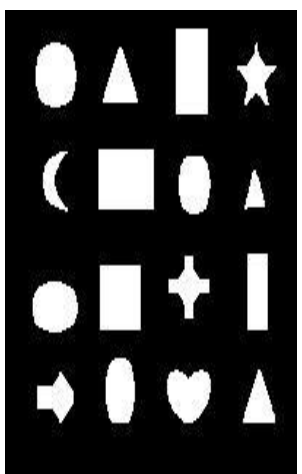

(b) Eroded image
Fig 7: Erosion Image 


\section{RESULTS}

Table 1. Implementation of Proposed Segmentation Approach
Original Image
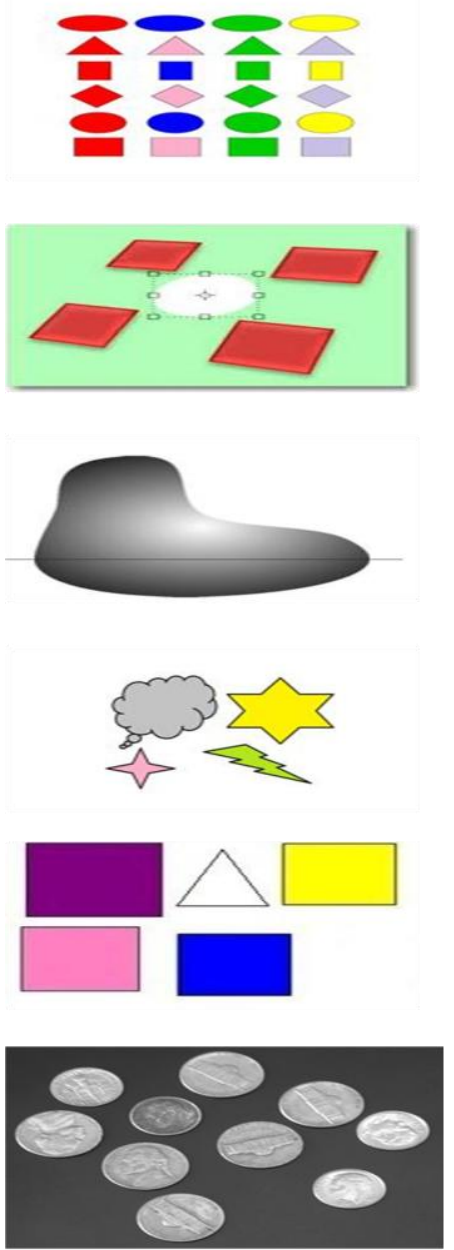

(a)

\section{Segmented Image}
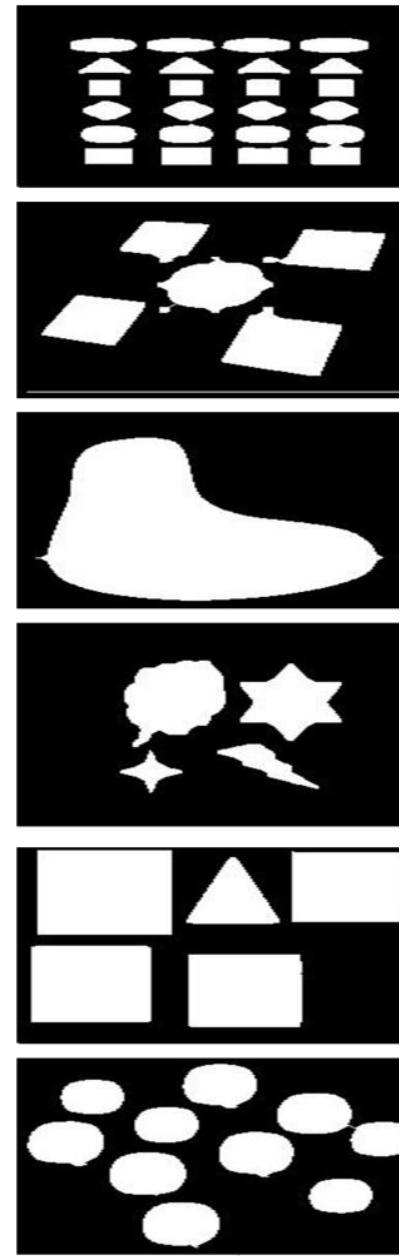

(b)
Table (I) shows the implementation of our proposed segmentation approach. Fig (a) shows the original image taken as an input and Fig (b) shows the final output of segmented image.

\section{CONCLUSION}

In this paper a new segmentation technique is proposed using morphological operations. In first step edge is detected using Fuzzy Canny method which can give better results compared to classical techniques of edge detection and in second stage, after edge detected, basic morphological operators are applied which are dilation and erosion and also flood fill is used to segment the image. It has been concluded that Segmentation using morphological basic operators can also segment the image. It is more simple and easy method than different segmentation methods.

\section{REFERENCES}

[1] Rafael C. Gonzalez, Richard E. Woods, "Digital Image Processing", 2nd ed., Beijing: Publishing House of Electronics Industry, 2007.
[2] R.C. Gonzalez and R.E. Woods, "Digital Image Processing", third edition, PHI publication, 2008.

[3] Dr. (Mrs.) G.Padmavathi, Dr.(Mrs.) P.Subashini and Mrs.A.Sumi "Empirical Evaluation of Suitable Segmentation Algorithms for IR Images", IJCSI International Journal of Computer Science Issues, Vol. 7, Issue 4, No 2, July 2010.

[4] Dhruven Prajapati, Jenish Gandhi, Kruti J. Dangarwala "A Comparative Study of various Image Segmentation Techniques", 2014 IJIRT | Volume 1 Issue 5 | ISSN: 2349-6002.

[5] D. Chudasama, T. Patel, S. Joshi, G. Prajapati "Survey on Various Edge Detection Techniques on Noisy Images" , IJERT International Journal of Engineering Research \& Technology ISSN: 2278-0181 Vol. 3 Issue 10, October- 2014

[6] Maini, Raman, and Himanshu Aggarwal, "Study and comparison of various image edge detection techniques", International Journal of Image Processing (IJIP), Issue 3, no. 1, Pp. 1-11, 2009.

[7] Er. Komal Sharma, Er. Navneet Kaur, "Comparative Analysis of Various Edge Detection Techniques", International Journal of Advanced Research in Computer Science and Software Engineering, Volume 3, Issue 12, December 2013.

[8] E. Sobel. Camera Models and Machine Perception.PhD thesis, Electrical Engineering Department, Stanford University, 1970

[9] J. M. S. Prewitt, "Object enhancement and extraction," in Picture Processing and Psychopictorics, B. Lipkin and A. Rosenfeld, Eds.New York: Academic, 1970, pp. 75-149 .

[10] J. F. Canny. "A computational approach to edge detection".IEEE Trans. Pattern Analysis and Machine Intelligence, vol. PAMI-8, no. 6, pp. 679-697, 1986.

[11] R. Adams, and L. Bischof, "Seeded region growing," IEEE Trans. Pattern Anal. Machine Intell., vol. 16, no. 6, pp. 641-647, June, 1994.

[12] S.L. Horowitz and T. Pavlidis, "Picture Segmentation by a Directed Split and Merge Procedure", Proc. ICPR, 1974, Denmark, pp.424-433.

[13] Ur Rehman Khan, K. Thakur "An Efficient Fuzzy Logic Based Edge Detection Algorithm for Gray Scale Image", International Journal of Emerging Technology and Advanced Engineering Website: www.ijetae.com (ISSN 2250-2459, Volume 2, Issue 8, August 2012)

[14] S. Patel, P.Trivedi, V. Gandhi and G. Prajapati, "2D Basic Shape Detection Using Region Properties" IJERT International Journal of Engineering Research \& Technology, Vol. 2 Issue 5, May-2013.

[15] Mrs. A. Borkar, Mr. M.Atulkumar "Detection of Edges Using Fuzzy Inference System”, International Journal of Innovative Research in Computer and Communication Engineering, Vol. 1, Issue 1, March 2013.

[16] T. Gajpal, Mr. S. Meshram "Edge Detection Technique Using Hybrid Fuzzy logic Method", IJERT Internationa Journal of Engineering Research \& Technology, Vol. 2 Issue 2, Febuary-2013.

[17] J. Serra, Image Analysis and Mathematical Morphology. New year Acadamic Press, 1982.

[18] M. L Comer, E. J. Delp "Morphological operations for color image processing" electronic imaging spiedigitallibrary.

[19] B. Baets, E. Kerre, M. Gupta "Fundamentals of Fuzzy Mathematical Morphology Part 1 Basic concepts" Overseas Publishers Association.

[20] R. Haralick and L. Shapiro Computer and Robot Vision, Vol. 1, Chap. 5, Addison-Wesley Publishing Company, 1992.

[21] A. Jain Fundamentals of Digital Image Processing, Prentice-Hall,1986,p3 